\title{
Heritability of pubertal timing: detailed evaluation of specific milestones in healthy boys and girls
}

\author{
Alexander S Busch ${ }^{1,2}$, Casper P Hagen ${ }^{1,2}$ and Anders Juul1,2 \\ ${ }^{1}$ Department of Growth and Reproduction, Rigshospitalet and 2International Center for Research and Research \\ Training in Endocrine Disruption of Male Reproduction and Child Health (EDMaRC), Rigshospitalet, University of \\ Copenhagen, Copenhagen, Denmark
}

Correspondence should be addressed to A S Busch

Email

alexander.siegfried.busch@ regionh.dk

\begin{abstract}
Objective: Pubertal timing is highly heritable. Observational studies were inconclusive concerning a potential sexspecific difference in the parental contribution, while genome-wide association studies highlighted a heterogeneity in the genetic architecture of pubertal timing between sexes. Our objectives were to evaluate the association of timing of pubertal milestones in offspring with parental pubertal timing and to identify the genetic basis of the heterogeneity. Design: (1.) Population-based mixed cross-sectional/longitudinal cohort (2006-2014, COPENHAGEN Puberty Study) comprising 1381 healthy Danish children including their parents. (2.) UK Biobank-based summary statistics of genetic data on timing of menarche $(n=188644)$, voice-break $(n=154459)$ and facial hair $(n=161470)$.

Methods: (1.) Participants underwent clinical examination(s) including blood sampling. Parental pubertal timing was obtained by questionnaire. Timing of milestones were analyzed using SAS-lifereg. (2.) Genetic correlations between pubertal outcomes were estimated using LD Score regression. Genetic heterogeneity was analyzed using METAL. Results: We observed significant associations of relative parental pubertal timing with timing of pubertal milestones in offspring of concordant sex, that is, fathers/sons (e.g. testicular enlargement: $P=0.004, \beta=0.34$ years per relative category) and mothers/daughters (e.g. thelarche: $P<0.001, \beta=0.45$ years per relative category). Fewer milestones were associated with relative parental pubertal timing in offspring of discordant sex compared to concordant sex. Large-scale genetic data highlight both moderate to strong genetic correlations between timing of menarche, voicebreak and facial hair. Out of 434 lead single-nucleotide polymorphisms significantly associated with at least one outcome, 39 exhibited a significant genetic heterogeneity between sexes $\left(P<1.15 \times 10^{-4}\right)$.

Conclusion: Our results highlight a distinct genetic heterogeneity of pubertal timing between sexes.
\end{abstract}

\section{Introduction}

Timing of puberty is highly heritable with both maternal and paternal genetic influences (1). While the association of maternal pubertal timing with pubertal timing in daughters is well established $(2,3)$, only sparse data exist on the association with pubertal timing in their sons. Further, little is known about the association of paternal pubertal timing with pubertal timing in both daughters and sons. Two recent observational studies suggested a shared parental contribution to pubertal timing in offspring $(3,4)$. However, observational studies have been underpowered to detect potential sex-specific differences in the association of parental pubertal timing with timing of puberty in offspring. At the same time, comparing female and male pubertal timing, recent genome-wide association studies (GWAS) revealed an overlapping genetic architecture of pubertal timing between sexes (5). Despite this overlap, results also highlighted a marked genetic heterogeneity of distinct genetic loci between

Published by Bioscientifica Ltd. 
sexes as well as a genetic heterogeneity between timing of different pubertal milestones within the same sex, for example, between thelarche and menarche in girls (6) and between voice-break and facial hair in boys (5).

Given the inconclusive evidence from observational studies on potential differences of the parental contribution to pubertal timing in offspring as well as evidence from large-scale genetic studies on distinct differences in the genetic architecture between male and female pubertal timing, we aimed to evaluate the associations of numerous pubertal milestones in offspring with parental pubertal timing. Further, we aimed to explore the heterogeneity in the genetic architecture of timing of pubertal milestones between sexes and identify genetic variants contributing to the genetic heterogeneity of pubertal timing between sexes.

\section{Patients and methods}

\section{Study populations}

Participants were recruited as part of the COPENHAGEN Puberty Study $(7,8)$, a population-based cohort study of healthy Danish children and adolescents (ClinicalTrials. gov ID: NCT01411527). Detailed information about the study has been described previously (8). In brief, the study is a mixed cross-sectional (Cohort A) (aged 6.121.9 (boys) and 5.8-23.4 (girls) years) and longitudinal (Cohort B) (age at baseline boys: 5.8-15.6 years, girls: $5.6-13.13$ years) study conducted at ten schools in the Copenhagen area from 2006-2014. A total of 3101 boys and 3102 girls were invited to participate. The overall participation rate was around $25 \%$ in boys and $35 \%$ in girls. Of 798 healthy boys and 1129 girls with available clinical pubertal staging, we excluded 239 boys and 308 girls due to non-Danish origin $(n=67 / 107)$, history of severe chronic illness $(n=2 / 3)$, age at inclusion $>19$ years $(n=19 / 33)$ or missing data on parental puberty $(n=150 / 165)$. After exclusion, data from 560 boys and 821 girls (cross-sectional study $n=487 / 757$; longitudinal study $n=73 / 64$ ) comprising $1247 / 1337$ visits were analyzed, respectively. Data on pubertal timing in both parents was available in $817 / 560$ parents of daughters/ boys, respectively. Single-nucleotide polymorphism (SNP) array data were not available for the COPENHAGEN Puberty Study.

\section{Clinical examinations and questionnaires}

Pubertal assessments in participants were performed by one of three male doctors (boys) or one of three female doctors (girls) who were trained by a pediatric endocrinologist (A $\mathrm{J})$. In boys, clinical examinations included assessment of puberty stage according to Tanner's classification (genitaland pubic-hair development by visual assessment) and assessment of testicular volume to the nearest millilitre by palpation using a Prader orchidometer (9). Testicular volume of $4 \mathrm{~mL}$ and above (at least one testis) was considered a marker of testicular enlargement, $\geq \mathrm{G} 2$ (scrotal enlargement and change in texture of the scrotal skin) defined gonadarche and $\geq \mathrm{PH} 2$ (sparse development of pigmented hair at the base of penis) defined pubarche. Sweat odor was recorded as a binary variable (yes/no) by self-reported characteristic change in body odor and assessment during examination. In case of incongruence, the latter was prioritized. Voice-break was defined as binary variable (yes/no) according to occurrence of unintentional falsetto notes or observed voice deepening during the examination. Self-reported occurrence was compared to observations during examinations and the latter was prioritized in case of incongruence. In girls, thelarche was assessed by palpation and pubarche was assessed by inspection according to Tanner's classification (10). Selfreported age at menarche was obtained by questionnaire. In both sexes, evaluation of axillary hair growth was performed by inspection. A wall-mounted stadiometer (Holtain Ltd., Crymych, UK) was used to measure standing height to the nearest $0.1 \mathrm{~cm}$. Participants were weighed on a digital electronic scale (Seca, Hamburg, Germany) to the nearest $0.1 \mathrm{~kg}$ while wearing light clothing and no shoes. BMI was calculated as weight divided by height squared $\left(\mathrm{kg} / \mathrm{m}^{2}\right)$. In parents, pubertal timing was obtained by questionnaire. Parents answered following questions: 'Did your puberty start early, average or late compared to your peers?' with the note: 'Puberty starts around the time when you experienced hair growth around your genitals and in your armpits' (fathers) and 'Puberty starts around the time when you experienced growth of your breasts and hair growth around your genitals and in your armpits' (mothers). Further, self-reported age at menarche in mothers was obtained by questionnaire. Paternity was not confirmed by genetic analysis.

\section{Reproductive hormone assays}

Non-fasting blood samples were drawn between $0800 \mathrm{~h}$ and $1400 \mathrm{~h}$, clotted and centrifuged; serum was stored at $-20^{\circ} \mathrm{C}$ until analyses. Serum levels of Luteinizing Hormone (LH) were measured by time-resolved immunofluorometric assays (Delfia; PerkinElmer) with a limit of detection (LOD) of $0.05 \mathrm{IU} / \mathrm{L}$. Intra- and interassay coefficients of variation 
(CVs) were $<5 \%$. Serum total testosterone was measured by a RIA using a Coat-A-Count RIA kit (Siemens) with a LOD of $0.23 \mathrm{nmol} / \mathrm{L}$. The assay was validated against LC-MS/MS in $n=20$ participants (11). Intra- and interassay CVs were $17 \%$ and $12.8 \%$, respectively. $\mathrm{LH}$ and total testosterone measurements below LOD were assigned a value of $0.025 \mathrm{IU} / \mathrm{L}$ and $0.115 \mathrm{nmol} / \mathrm{L}$, respectively $(0.5$ times the LOD). The median (range) age at blood sampling for participants that were included into the study: crosssectional part (Cohort A) 10.7 (6.1-18.6) years in boys and 11.1 (5.8-19.0) years in girls; longitudinal (Cohort B) 11.7 (6.1-16.4) years in boys and 11.4 (5.9-16.5) years in girls.

\section{GWAS on pubertal timing}

GWAS summary statistic for relative timing of voice-break $(n=154459)$, relative timing of facial hair $(n=161470)$ and age at menarche $(n=188$ 644) were obtained from the Neale Lab (http://www.nealelab.is/uk-biobank/). Genotyping, quality control procedures and analysis model have been described previously $(12,13)$. Relative age at voice-break and facial hair were obtained by touchscreen questionnaire (Question: 'When did your voice break?' and 'When did you start to grow facial hair?'; Answers: 'younger than average, about average, older than average, do not know, prefer not to answer'). Age at menarche in years was also obtained by touchscreen questionnaire (Question: 'How old were you when your periods started?'). Genotype and phenotype data from the UK Biobank have previously been analysed and published including data on menarche, voice-break, and facial hair that was included in previous $\operatorname{GWAS}(5,14)$. Genetic correlations $\left(r_{g}\right)$ between the traits were calculated using LD Score Regression $(15,16)$. GWAS summary statistics were analyzed and annotated using FUMA SNP2GENE (17). Heterogeneity between puberty measures for SNP associations were quantified using METAL $\mathrm{I}^{2}$ statistics under the default approach (18). Genetic correlations based on UK Biobank data have been published previously as part of a data resource $(13,19)$ as well as original articles $(5,14,20)$.

\section{Statistical analysis}

We performed analyses (SAS: PROC lifereg, normal distribution) to estimate the mean age (95\% CI) at pubertal milestones (21). PROC lifereg fits parametric regression models to left-/right-/and interval-censored survival data using maximum likelihood estimation. Children achieving a pubertal milestone during follow-up were included as interval-censored data, while data were included as right censored data if a child had not achieved the milestone at his/her last examination (or only examination if seen once) and included as left censored data if a child had already achieved the milestone at his/ her first examination (or only examination if seen once). This model takes the broad age range of the cohort into account and underweights outliers. While the exact age at which the milestone has been achieved remains unknown, the estimates represent a corresponding age span. Parental pubertal timing (early/average/late) and age at menarche in mothers were introduced into the model as continuous variable. The PROBPLOT statement was used for visual assessment of goodness-of-fit and Turnbull estimates of the cumulative distribution functions approximated a straight line on an inverse distribution scale (data not shown). Interaction between parental pubertal timing was tested via the MODEL statement. A $P$-value of $<0.05$ was considered statistically significant. Correcting for multiple testing (434 lead SNPs), the $\mathrm{P}_{\text {Heterogeneity }}$ threshold was set to $<1.15 \times 10^{-4}$. A lead SNP was defined as independent SNP at genome-wide significance with a pairwise $r^{2}<0.1$.

\section{Ethical considerations}

The COPENHAGEN Puberty Study (ClinicalTrials.govID: NCT01411527) was approved by the ethical committee of The Capital Region of Denmark (No. KF01282214) as well as the Danish Data Protection Agency (No. 2015-414494). Written informed consent was obtained from all parents or guardians of the children and adolescents.

\section{Results}

We observed significant associations of self-reported parental pubertal timing with timing of all pubertal milestones in offspring of concordant sex, that is, mothers and daughters as well as father and sons (Fig. 1 and Table 1). Effect sizes in Fig. 1 and Table 1 are presented as years per relative category of parental pubertal timing, meaning that timing of pubertal milestones in offspring of a parent with early or late pubertal timing (compared average pubertal timing) occurs earlier, that is, minus the effect size, or later, that is, plus the effect size, respectively. Further, selfreported age at menarche in mothers was associated with all pubertal milestones in daughters, except axillary hair growth (Table 2). Concerning associations with pubertal timing in offspring of discordant sex, that is, mother/sons and father/daughter, the results were heterogeneous: While 


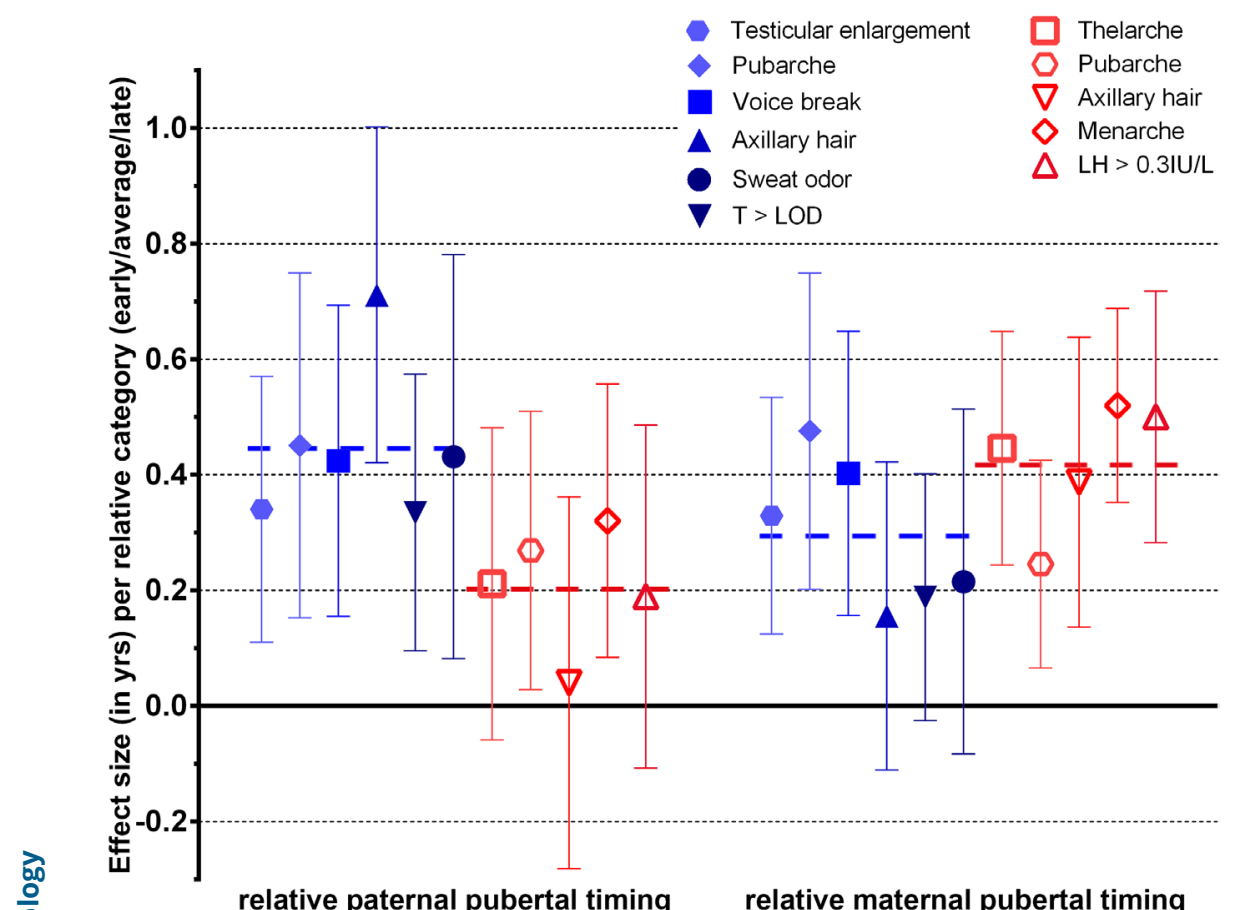

\section{Figure 1}

Parental pubertal timing and timing of pubertal milestones in offspring. Timing of pubertal milestones in sons (blue) and daughters (red). Symbols correspond to pubertal milestones. Vertical bars extend to $95 \% \mathrm{Cl}$. The left panel shows associations with pubertal timing in fathers and the left panel with pubertal timing in mothers. Dashed lines indicate mean effect estimates of the association of pubertal milestones in sons and daughters with pubertal timing in fathers and mothers, respectively. pubertal timing in fathers was significantly associated with timing of pubarche and menarche in daughters, it was not associated with timing of thelarche or axillary hair growth. Pubertal timing in mothers was significantly associated with timing of testicular enlargement, pubarche and voice-break in sons, but not with timing of sweat odor or axillary hair growth. Self-reported age at menarche in mothers was significantly associated with all pubertal milestones in sons, except sweat odor (Table 2). We further included hormonal pubertal outcomes in our analysis, that is, testosterone above LOD $(0.23 \mathrm{nmol} / \mathrm{L})$ in boys and $\mathrm{LH}>0.3 \mathrm{IU} / \mathrm{L}$ in girls, representing crude hormonal indicators of pubertal onset $(11,22)$. We observed a similar pattern of significant associations in concordant sex and no associations in discordant sex for hormonal pubertal outcomes (Table 1). Interaction analysis between parental pubertal timing and timing in offspring revealed a nominal significant interaction between paternal and maternal pubertal timing on 1.) male pubarche $(P=0.02)$ and (2.) female axillary hair growth $(P=0.002)$. After

Table 1 Parental pubertal timing and timing of pubertal milestones in offspring.

\begin{tabular}{|c|c|c|c|c|c|c|}
\hline \multirow[b]{2}{*}{ Pubertal events } & \multicolumn{3}{|c|}{ Relative paternal pubertal timing } & \multicolumn{3}{|c|}{ Relative maternal pubertal timing } \\
\hline & $n$ & Estimate $^{a}$ (years per category) & $P$-value & $n$ & Estimate $^{a}$ (years per category) & $P$-value \\
\hline \multicolumn{7}{|l|}{ Male pubertal milestones } \\
\hline Testicular enlargement ${ }^{\mathrm{b}}$ & 446 & $0.34(0.11$ to 0.57$)$ & 0.004 & 541 & $0.33(0.12$ to 0.53$)$ & 0.002 \\
\hline Pubic hair & 444 & $0.45(0.15$ to 0.75$)$ & 0.003 & 539 & $0.48(0.20$ to 0.75$)$ & 0.001 \\
\hline Voice break & 391 & $0.42(0.16$ to 0.69$)$ & 0.002 & 479 & $0.40(0.16$ to 0.65$)$ & 0.001 \\
\hline Sweat odor & 446 & $0.43(0.08$ to 0.78$)$ & 0.016 & 542 & $0.22(-0.08$ to 0.51$)$ & 0.158 \\
\hline Axillary hair & 446 & $0.71(0.42$ to 1.00$)$ & $<0.001$ & 543 & $0.16(-0.11$ to 0.42$)$ & 0.252 \\
\hline Total testosterone above LOD & 422 & $0.33(0.10$ to 0.57$)$ & 0.006 & 507 & $0.19(-0.03$ to 0.4$)$ & 0.084 \\
\hline mean & - & $0.45(0.20$ to 0.69$)$ & - & - & $0.30(0.07$ to 0.52$)$ & - \\
\hline \multicolumn{7}{|l|}{ Female pubertal milestones } \\
\hline Thelarche & 622 & $0.21(-0.06$ to 0.48$)$ & 0.125 & 783 & $0.45(0.24$ to 0.65$)$ & $<0.001$ \\
\hline Pubic hair & 616 & $0.27(0.03$ to 0.51$)$ & 0.029 & 776 & $0.25(0.07$ to 0.43$)$ & 0.007 \\
\hline Axillary hair & 580 & $0.04(-0.28$ to 0.36$)$ & 0.806 & 737 & $0.39(0.14$ to 0.64$)$ & 0.003 \\
\hline Menarche & 628 & $0.32(0.08$ to 0.56$)$ & 0.008 & 791 & $0.52(0.35$ to 0.69$)$ & $<0.001$ \\
\hline LH above 0.3 IU/L & 532 & $0.19(-0.11$ to 0.49$)$ & 0.211 & 674 & $0.50(0.28$ to 0.72$)$ & $<0.001$ \\
\hline mean & - & $0.21(0.02$ to 0.39$)$ & - & - & $0.42(0.23$ to 0.61$)$ & - \\
\hline
\end{tabular}

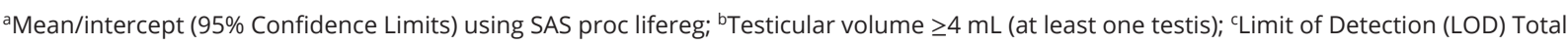
testosterone: $0.23 \mathrm{nmol} / \mathrm{L}$. 
Table 2 Maternal age at menarche and timing of pubertal milestones in offspring.

\begin{tabular}{|c|c|c|c|}
\hline \multirow[b]{2}{*}{ Pubertal events } & \multicolumn{3}{|c|}{ Maternal age at menarche } \\
\hline & $n$ & $\begin{array}{l}\text { Estimate }^{\text {(years per }} \\
\text { year difference of age } \\
\text { at menarche) }\end{array}$ & $P$-value \\
\hline \multicolumn{4}{|c|}{ Male pubertal milestones } \\
\hline $\begin{array}{l}\text { Testicular } \\
\text { enlargement }{ }^{\mathrm{b}}\end{array}$ & 506 & 0.21 (0.09 to 0.32$)$ & $<0.001$ \\
\hline Pubic hair & 506 & $0.19(0.03$ to 0.34$)$ & 0.017 \\
\hline Voice break & 445 & $0.19(0.05$ to 0.33$)$ & 0.008 \\
\hline Sweat odor & 507 & $0.10(-0.06$ to 0.27$)$ & 0.228 \\
\hline Axillary hair & 507 & $0.16(0.01$ to 0.30$)$ & 0.035 \\
\hline $\begin{array}{l}\text { Total testosterone } \\
\text { above LOD }\end{array}$ & 473 & 0.15 (0.03 to 0.27$)$ & 0.018 \\
\hline \multicolumn{4}{|c|}{ Female pubertal milestones } \\
\hline Thelarche & 758 & $0.20(0.08$ to 0.33$)$ & 0.001 \\
\hline Pubic hair & 752 & $0.18(0.08$ to 0.29$)$ & 0.001 \\
\hline Axillary hair & 712 & $0.10(-0.05$ to 0.25$)$ & 0.190 \\
\hline Menarche & 766 & 0.29 (0.20 to 0.39$)$ & $<0.001$ \\
\hline LH above $0.3 \mathrm{IU} / \mathrm{L}$ & 648 & $0.23(0.10$ to 0.36$)$ & 0.001 \\
\hline
\end{tabular}

aMean/intercept (95\% Confidence Limits) using SAS proc lifereg;

bTesticular volume $\geq 4 \mathrm{~mL}$ (at least one testis); CLimit of Detection (LOD)

Total testosterone: $0.23 \mathrm{nmol} / \mathrm{L}$.

correction for multiple testing (eleven tests), only female axillary hair growth remained significant $\left(P<4.5 \times 10^{-3}\right)$. All other outcomes did not interact significantly.

Using publicly available large-scale genetic data from the UK Biobank study, we confirmed moderate to strong genetic correlations $\left(\mathrm{r}_{\mathrm{g}}\right.$ ) between relative timing of voicebreak, relative timing of facial hair in men and age at menarche in women, that is, $r_{g}$ (s.E.) facial hair vs voicebreak: 0.86 (0.02); $\mathrm{r}_{\mathrm{g}}$ facial hair vs menarche: 0.55 (0.02); $r_{g}$ voice-break vs menarche: $0.70(0.03)$. Four hundred and thirty-four lead SNPs were significantly associated with timing of menarche and/or voice-break and/or facial hair. Of these, 39 lead SNPs exhibited a significant genetic heterogeneity between sexes $\left(\mathrm{P}_{\text {Heterogeneity }}<1.15 \times 10^{-4}\right.$ ) (Supplementary Fig. 1 and Supplementary Tables 1, 2, see section on supplementary materials given at the end of this article). Thirty-five of the 39 lead SNPs exhibited a significant heterogeneity between sexes, that is, between age at menarche and the two male puberty outcomes but not between voice-break and facial hair. Among these were rs3767357-RXRG (genome-wide significant associated with age at menarche but neither with relative timing of voice-break nor with relative timing of facial hair) as well as rs1516883-TMEM38B and rs9391253-LIN28B (genomewide significantly associated with timing of all three milestones). Four of the 39 lead SNPs, that is, rs1345417SOX2, rs10954315-MKLN1, rs12913832-HERC2 and rs1129038-HERC2, exhibited significant heterogeneity between all three puberty measures. Notably, none of the SNPs that exhibited significant genetic heterogeneity were located on the X Chromosome.

\section{Discussion}

In this comprehensive analysis of observational data, we demonstrate that self-reported pubertal timing in parents is consistently associated with timing of pubertal milestones in offspring of concordant sex. At the same time, there is a lesser degree of congruence in the association with discordant sex. Based on these clinical observations, our results point to a potential heterogeneity in the parental contribution to the pubertal timing in their offspring. Mirroring these clinical observations, large-scale genetic data underlines the overlapping genetic architecture between males and females but also highlights the distinct loci exhibiting significant genetic heterogeneity between sexes.

Both maternal and paternal genetic information is thought to shape the heritability of pubertal timing in an additive manner. Previous studies using self-reported relative pubertal timing or family history of puberty disorder pointed to a shared contribution of both sexes to pubertal timing in healthy offspring as well as to the propensity of puberty disorders, for example, Constitutional Delay of Growth and Puberty (CDGP) (3, 23), respectively. However, a potential heterogeneity in the contribution between sexes remained speculative. This is particularly due to the scarcity of data on paternal pubertal timing. One recent study, including both paternal and maternal puberty data, reported subtle differences between the association of parental pubertal timing with pubertal milestones in offspring: Age at menarche in daughters but not age at thelarche and pubarche was strongly associated with parental pubertal timing. Further, timing of pubarche and menarche but not thelarche in daughters was associated with pubertal timing of fathers (3).

In our large observational study, we extend previous observations by including numerous clinically assessed pubertal milestones in offspring along with maternal as well as paternal pubertal timing. We show that parental pubertal timing is consistently associated with all pubertal milestones in offspring of the concordant sex. However, concerning associations in discordant sex, results point to a lesser degree of congruence. This includes a higher mean effect estimate of the association with pubertal milestones in offspring of concordant sex compared to discordant sex. 
A comparable sex-dimorphic pattern of effect estimates, at least concerning maternal pubertal timing and timing in offspring, has been reported in a large Danish cohort (4).

A potential explanation for this discrepancy in the association between paternal and maternal timing with timing of pubertal milestones in offspring includes nonadditive genetic effects. However, only one out of 11 pubertal milestones, that is, axillary hair growth in girls, exhibited evidence of a significant interaction between parental pubertal timing. In light of the predominantly additive effect of male and female pubertal timing on timing of pubertal milestones in offspring, this potentially points to an alternative explanation for our observations: a heterogeneity in the genetic architecture of male and female puberty.

In order to explore the genetic architecture of male and female puberty, we chose to use data from the UK Biobank study, a public resource with deep phenotyping and genomic data from a population of close genetic distance to our Danish clinical cohort $(24,25)$. In contrast to our observational data, large-scale genetic data on pubertal timing was only available on few pubertal milestones, that is, menarche in women and facial hair and voice-break in men. In our analysis, we quantify the overlap and confirm a moderate to strong genome-wide genetic correlation between timing of male and female pubertal milestones, that is, between facial hair and voice-break in men on the one side and timing of menarche in women on the other side. Previous largescale genetic data already highlighted a heterogeneity in the genetic architecture of male and female puberty using partially overlapping datasets $(5,20)$. When comparing results on overlapping traits of our observational data and large-scale genetic data, that is, timing of voice-break and menarche, we see that the moderate to strong genetic correlation between the traits based on large-scale genetic data is mirrored by the significant associations of paternal pubertal timing with age at menarche in daughters as well as maternal pubertal timing with age at voice-break in sons in the observational data. At the same time, we observed differences in the effect sizes of other milestones in offspring of discordant sex, for example, paternal pubertal timing and timing of thelarche in daughters. We speculate that the differences in associations between a single milestone in one sex and multiple milestones in the other sex might be indicative of a heterogeneity between pubertal milestones within the same sex. In the large-scale genetic dataset, this is directly highlighted for the male part by the imperfect genetic correlation between the two males milestones, that is, facial hair and voice-break, and indirectly highlighted for the female part by the significant differences in genetic correlation of voice-break and menarche vs facial hair and menarche. At least in females this heterogeneity between milestones within the same sex has previously been reported from a twin study in girls that pointed to strong but imperfect genetic correlations between breast development, pubic-hair development and menarche (26). However, in lack of large-scale genetic data on all pubertal milestones, we are unable to delineate or quantify the heterogeneity between sexes and pubertal milestones within the same sex.

At last, the question remains which specific genes contribute to the heterogeneity of pubertal timing between sexes by exhibiting a sex-specific penetrance, that is, which genetic loci are of significantly more importance for one sex compared to the other. We identified 434 lead SNPs, largely published previously (5, 14), that were associated with timing of at least one of the puberty outcomes. Using the three puberty outcomes in a triangle set-up, we identify SNPs exhibiting heterogeneity between male and female puberty, that is, SNPs that exhibit heterogeneity between timing of voice-break and female puberty outcomes but not between the two male pubertal outcomes, that is, facial hair and voice-break. Implied genes with a potentially differential role in male and female pubertal timing includes $R X R G$ (femalespecific) coding for a retinoic $X$ receptor that exhibits a sex-specific expression pattern in the mouse pituitary (27). Further signals adjacent to LIN28B and TMEM38B exhibit a genetic heterogeneity pointing to a more significant role for female pubertal timing compared to male pubertal timing. Both genes are implicated in co-regulation of BMI and puberty timing (14). Four signals exhibit a genetic heterogeneity between all three pubertal milestones. These loci are linked to SOX2, MKLN1 and HERC2, known for their role in hair growth and hair color $(28,29)$. Based on the concept that puberty is initiated centrally and separates into several endpoints, that is, milestones, we speculate that genetic variants linked to central processes, for example, neurodevelopment including GnRH neuron migration or pituitary development, contribute to the heterogeneity between sexes, while other genetic variants linked to the peripheral development of distinct pubertal milestones contribute to the heterogeneity between milestones within the same sex. This has previously been shown for SNPs linked to $L I N 28 B$, involved in general cell proliferation, and $F S H B$, involved specific signal transmission from the pituitary gonadal target tissues $(14,30)$. 
Potential limitations include that self-reported relative pubertal timing in parents represents a subjective variable comprising a combination of different recallable pubertal milestones. However, in adult women, the validity of both recalled pubertal timing as well as age of menarche have been demonstrated previously $(31,32)$. Such studies on recalled pubertal timing in adult men, that is, voice-break or facial hair, do not exist. However, concerning timing of voice-break, a recent comparison of two genetic studies reporting relative (early/average/late) and binned (bins of 2 years) timing have been shown to exhibit a strong genetic correlation $\left(r^{2} \approx 0.8\right)$ highlighting the validity our approach (5). Most importantly, under the assumption that self-reported relative pubertal timing in parents of concordant and discordant sex is independent of the clinical puberty staging in offspring, the significant associations of pubertal outcomes in offspring with parental pubertal timing indicate that the risk of bias, particularly concerning bias between sexes, is low. In addition, some of the clinically assessed outcomes in children might be more objective, for example, testicular volume, thelarche, pubarche, axillary hair or menarche, compared to others, for example, voicebreak or sweat odor, potentially introducing a bias. The low participation rate of $25-35 \%$ in the COPENHAGEN Puberty Study implies the risk of selection bias. However, again we do not believe that this potential source of bias confounds the association of parental pubertal timing with pubertal timing in offspring, that is, the heritability of pubertal timing. Further, fatherhood was not proved by genetic analysis. However, we assume that this fact did not introduce a significant bias. Finally, it is important to highlight that our results exclusively compare the relative variation of pubertal onset in both sexes and not absolute differences. For reasons still to be elucidated, sexual maturation starts around 1 year earlier in girls compared to boys (33).

Our results highlight the heterogeneity of pubertal timing between sexes as well as potential heterogeneity between pubertal milestones within the same sex using both observational as well as large-scale genetic data.

\section{Supplementary materials}

This is linked to the online version of the paper at https://doi.org/10.1530/ EJE-20-0023.

\section{Declaration of interest}

The authors declare that there is no conflict of interest that could be perceived as prejudicing the impartiality of this study.

\section{Funding}

This work was supported by Danish Agency for Science, Technology and Innovation (09-067180); Danish Ministry of the Environment, CeHoS (MST621-00065); Capital Region of Denmark (R129-A3966); Ministry of Higher Education and Science (DFF-1331-00113); Innovation Fund Denmark (InnovationsFonden, grant no. 14-2013-4) and The International Center for Research and Research Training in Endocrine Disrupting Effects of Male Reproduction and Child Health (EDMaRC).

\section{Acknowledgements}

The authors are grateful for the technical help from the skilled personnel in our lab as well as the participating children, adults and their families.

\section{References}

1 Silventoinen K, Haukka J, Dunkel L, Tynelius P \& Rasmussen F. Genetics of pubertal timing and its associations with relative weight in childhood and adult height: the Swedish Young Male Twins Study. Pediatrics 2008121 e885-e891. (https://doi.org/10.1542/peds.20071615)

2 Malina RM, Ryan RC \& Bonci CM. Age at menarche in athletes and their mothers and sisters. Annals of Human Biology 199421 417-422. (https://doi.org/10.1080/03014469400003432)

3 Wohlfahrt-Veje C, Mouritsen A, Hagen CP, Tinggaard J, Mieritz MG, Boas M, Petersen JH, Skakkebæk NE \& Main KM. Pubertal onset in boys and girls is influenced by pubertal timing of both parents. Journal of Clinical Endocrinology and Metabolism 2016101 2667-2674. (https://doi.org/10.1210/jc.2016-1073)

4 Brix N, Ernst A, Lauridsen LLB, Parner E, Støvring H, Olsen J, Henriksen TB \& Ramlau-Hansen CH. Timing of puberty in boys and girls: a population-based study. Paediatric and Perinatal Epidemiology 201933 70-78. (https://doi.org/10.1111/ppe.12507)

5 Hollis B, Day FR, Busch AS, Thompson DJ, Soares ALG, Timmers PRHJ, Kwong A, Easton DF, Joshi PK, Timpson NJ et al. Genomic analysis of male puberty timing highlights shared genetic basis with hair colour and lifespan. Nature Communications 202011 1536. (https://doi.org/10.1038/s41467-020-14451-5)

6 Busch AS, Hagen CP, Main KM, Pereira A, Corvalan C, Almstrup K, Mericq V \& Juul A. Genetic variation of follicle-stimulating hormone action is associated with age at testicular growth in boys. Journal of Clinical Endocrinology and Metabolism 2017102 1740-1749. (https:// doi.org/10.1210/jc.2016-4013)

7 Aksglaede L, Sørensen K, Petersen JH, Skakkebaek NE \& Juul A. Recent decline in age at breast development: the Copenhagen Puberty Study. Pediatrics 2009123 e932-e939. (https://doi. org/10.1542/peds.2008-2491)

8 Sørensen K, Aksglaede L, Petersen JH \& Juul A. Recent changes in pubertal timing in healthy Danish boys: associations with body mass index. Journal of Clinical Endocrinology and Metabolism 201095 263-270. (https://doi.org/10.1210/jc.2009-1478)

9 Marshall WA \& Tanner JM. Variations in the pattern of pubertal changes in boys. Archives of Disease in Childhood 197045 13-23. (https://doi.org/10.1136/adc.45.239.13)

10 Marshall WA \& Tanner JM. Variations in pattern of pubertal changes in girls. Archives of Disease in Childhood 196944 291-303. (https:// doi.org/10.1136/adc.44.235.291)

11 Mouritsen A, Søeborg T, Johannsen TH, Aksglaede L, Sørensen K, Hagen CP, Mieritz MG, Frederiksen H, Andersson AM \& Juul A. Longitudinal changes in circulating testosterone levels determined by LC-MS/MS and by a commercially available radioimmunoassay in healthy girls and boys during the pubertal transition. Hormone Research in Paediatrics 201482 12-17. (https://doi. org/10.1159/000358560) 
12 Bycroft C, Freeman C, Petkova D, Band G, Elliott LT, Sharp K, Motyer A, Vukcevic D, Delaneau O, O'Connell J et al. The UK Biobank resource with deep phenotyping and genomic data. Nature 2018562 203-209. (https://doi.org/10.1038/s41586-018-0579-z)

13 Overview of the data QC, code, and GWAS summary output from the 2017 UK Biobank data release. (available at: https:/github.com/ Nealelab/UK_Biobank_GWAS)

14 Day FR, Thompson DJ, Helgason H, Chasman DI, Finucane H, Sulem P, Ruth KS, Whalen S, Sarkar AK, Albrecht E et al. Genomic analyses identify hundreds of variants associated with age at menarche and support a role for puberty timing in cancer risk. Nature Genetics 201749 834-841. (https://doi.org/10.1038/ng.3841)

15 Bulik-Sullivan BK, Loh PR, Finucane HK, Ripke S, Yang J, Schizophrenia Working Group of the Psychiatric Genomics, Patterson N, Daly MJ, Price AL \& Neale BM. LD Score regression distinguishes confounding from polygenicity in genome-wide association studies. Nature Genetics 201547 291-295. (https://doi. org/10.1038/ng.3211)

16 Zheng J, Erzurumluoglu AM, Elsworth BL, Kemp JP, Howe L, Haycock PC, Hemani G, Tansey K, Laurin C, Early Genetics and Lifecourse Epidemiology (EAGLE) Eczema Consortium et al. LD Hub: a centralized database and web interface to perform LD score regression that maximizes the potential of summary level GWAS data for SNP heritability and genetic correlation analysis. Bioinformatics 201733 272-279. (https://doi.org/10.1093/bioinformatics/btw613)

17 Watanabe K, Taskesen E, van Bochoven A \& Posthuma D. Functional mapping and annotation of genetic associations with FUMA. Nature Communications 20178 1826. (https://doi.org/10.1038/s41467-01701261-5)

18 Willer CJ, Li Y \& Abecasis GR. METAL: fast and efficient metaanalysis of genomewide association scans. Bioinformatics 201026 2190-2191. (https://doi.org/10.1093/bioinformatics/btq340)

19 Genetic correlation between traits and disorders in the UK Biobank. (available at: https://ukbb-rg.hail.is/)

20 Day FR, Bulik-Sullivan B, Hinds DA, Finucane HK, Murabito JM, Tung JY, Ong KK \& Perry JRB. Shared genetic aetiology of puberty timing between sexes and with health-related outcomes. Nature Communications 20156 8842. (https://doi.org/10.1038/ncomms9842)

21 Aksglaede L, Olsen LW, Sørensen TIA \& Juul A. Forty years trends in timing of pubertal growth spurt in 157,000 Danish school children. PLOS ONE 20083 e2728. (https://doi.org/10.1371/journal. pone.0002728)

22 Kaplowitz P, Bloch C \& Section on Endocrinology, American Academy of Pediatrics. Evaluation and referral of children with signs of early puberty. Pediatrics 2016137 e20153732. (https://doi. org/10.1542/peds.2015-3732)

23 Wehkalampi K, Widén E, Laine T, Palotie A \& Dunkel L. Patterns of inheritance of constitutional delay of growth and puberty in families of adolescent girls and boys referred to specialist pediatric care. Journal of Clinical Endocrinology and Metabolism 200893 723-728. (https://doi.org/10.1210/jc.2007-1786)

24 Novembre J, Johnson T, Bryc K, Kutalik Z, Boyko AR, Auton A, Indap A, King KS, Bergmann S, Nelson MR et al. Genes mirror geography within Europe. Nature 2008456 98-101. (https://doi. org/10.1038/nature07331)

25 Bycroft C, Freeman C, Petkova D, Band G, Elliott LT, Sharp K, Motyer A, Vukcevic D, Delaneau O, O'Connell J et al. The UK Biobank resource with deep phenotyping and genomic data. Nature 2018562 203-209. (https://doi.org/10.1038/s41586-018-0579-z)

26 van den Berg SM, Setiawan A, Bartels M, Polderman TJC, van der Vaart AW \& Boomsma DI. Individual differences in puberty onset in girls: bayesian estimation of heritabilities and genetic correlations. Behavior Genetics 200636 261-270. (https://doi.org/10.1007/s10519005-9022-y)

27 Hou H, Uusküla-Reimand L, Makarem M, Corre C, Saleh S, Metcalf A, Goldenberg A, Palmert MR \& Wilson MD. Gene expression profiling of puberty-associated genes reveals abundant tissue and sex-specific changes across postnatal development. Human Molecular Genetics 201726 3585-3599. (https://doi.org/10.1093/hmg/ddx246)

28 Clavel C, Grisanti L, Zemla R, Rezza A, Barros R, Sennett R, Mazloom AR, Chung CY, Cai X, Cai CL et al. Sox2 in the dermal papilla niche controls hair growth by fine-tuning BMP signaling in differentiating hair shaft progenitors. Developmental Cell 201223 981-994. (https://doi.org/10.1016/j.devcel.2012.10.013)

29 Morgan MD, Pairo-Castineira E, Rawlik K, Canela-Xandri O, Rees J, Sims D, Tenesa A \& Jackson IJ. Genome-wide study of hair colour in UK Biobank explains most of the SNP heritability. Nature Communications 20189 5271. (https://doi.org/10.1038/s41467-01807691-z)

30 Busch AS, Hagen CP, Assens M, Main KM, Almstrup K \& Juul A. Differential impact of genetic loci on age at thelarche and menarche in healthy girls. Journal of Clinical Endocrinology and Metabolism 2018 103 228-234. (https://doi.org/10.1210/jc.2017-01860)

31 Casey VA, Dwyer JT, Coleman KA, Krall EA, Gardner J \& Valadian I. Accuracy of recall by middle-aged participants in a longitudinal study of their body size and indices of maturation earlier in life. Annals of Human Biology 199118 155-166. (https://doi. org/10.1080/03014469100001492)

32 Koo MM \& Rohan TE. Accuracy of short-term recall of age at menarche. Annals of Human Biology 199724 61-64. (https://doi. org/10.1080/03014469700004782)

33 Parent AS, Teilmann G, Juul A, Skakkebaek NE, Toppari J \& Bourguignon JP. The timing of normal puberty and the age limits of sexual precocity: variations around the world, secular trends, and changes after migration. Endocrine Reviews 200324 668-693. (https:// doi.org/10.1210/er.2002-0019)

Received 9 January 2020

Revised version received 25 April 2020

Accepted 29 April 2020 